\title{
FuZZY BASED HyPERSPECTRAL IMAGE Segmentation Using Subpixel Detection
}

\author{
VeeraSenthilKumar.G ${ }^{1}$, Dhivya. $M^{2}$, Sivasangari. ${ }^{3}$, Suganya. $\mathrm{V}^{4}$ \\ ${ }^{1}$ Assistant Professor, ${ }^{2}$ UG Student, ${ }^{3}$ UG Student, ${ }^{4}$ UG Student \\ Department of ECE,Velammal College of Engineering and Technology, Tamil Nadu, \\ India
}

\begin{abstract}
Spatial analysis of images sensed and captured from a satellite provides less adequate information about a remote location. Hence spectral analysis becomes essential. Hyperspectral image is one of the remotely sensed images, superior to multispectral images in providing spectral information. Target detection is one of the significant requirements in many areas such as military, agriculture etc. Sub pixel target detection, which further divides each pixel of the image into partitions, is possible only with spectral analysis of hyperspectral image. This paper focuses on developing an algorithm for segmenting hyperspectral image using sub pixel target detection followed by Fuzzy C-Means(FCM) clustering technique. Principal Component Analysis (PCA) is the basic step adopted to reduce the high dimensional data of image to low dimensional data. Mixture tuned matched filtering technique is used for sub pixel target detection because it is a combination of linear spectral unmixing and matched filtering and has advantages of both the techniques.
\end{abstract}

\section{Keywords}

Spectral analysis, Hyperspectral image segmentation, Sub-pixel detection and FCM.

\section{INTRODUCTION}

HYPERSPECTRAL image is a high-dimensional image set that typically consists of 100-200 image channels. Hyper spectral imaging is the simultaneous acquisition of hundreds of narrow contiguous spectral bands. Multispectral images consists only RGB colors and captures image data at specific frequencies across the electromagnetic spectrum. Each channel in the hyperspectral image is a gray scale image that indicates the spectral response to a particular frequency in the electromagnetic spectrum. These frequencies usually include the visible spectrum of light, but almost all of the channels are focused in the infrared range (IR). This allows a hyperspectral image to have features that are invisible in a standard color image. Each pixel in the hyperspectral image will possess a spectral response vector that is the highdimensional equivalent of the 'color' of the pixel. Certain materials have a specific spectral signature which can be used to identify pixels containing that material. For this reason, there is great demand in developing fast detection techniques in hyperspectral imaging for applications such as Aerial surveillance, agricultural and chemical analysis, mineral analysis and medical imaging. These hyperspectral images are acquired using hyperspectral sensors. There are varieties of sensors available for capturing these images. Important of these are AVIRIS and HYDICE. The AVIRIS sensor provides data sets of the hyperspectral images consisting of multiple bands. Datasets of AVIRIS sensor have been used in this project for experimentation. Hyperspectral

DOI : $10.5121 /$ ijist.2014.4322 
images are mainly used in three areas of interest namely Anomaly detection, Target detection and Material identification. This paper focuses on target detection i.e., finding within the imagery, materials that are present and match them with the existing spectral libraries. All pixels whose spectra match the target spectrum are marked as potential targets. The underlying assumption is that the pixels containing the target are all "pure".

Jun $\mathrm{Li}$ et al. proposed an algorithm for supervised Hyperspectral image segmentation using Bayesian framework with active learning in the year 2011. The algorithm implements two main steps semi supervised learning of the posterior class distributions followed by segmentation, which infers an image of class labels from a posterior distribution built on the learned class distributions and on a Markov random field [2]. This method is about integration of subspace projection methods with the MLR algorithm, combined with the use of spatial-contextual information [1]. Janete S. Borges et al. proposed a supervised technique to segment hyperspectral images: the Bayesian segmentation based on discriminative classification and on multilevel logistic (MLL) spatial prior [3]. Hernâni Gonçalves et al. proposed AIR method which is based on the combination of image segmentation and SIFT, complemented by a robust procedure of outlier removal. This combination allows for an accurate obtention of tie points for a pair of remote sensing images, being a powerful scheme for AIR [4]. Gokan Bilgin et al. presented an unsupervised Hyperspectral image segmentation with a new subtractive-clustering-based similarity segmentation and a novel cluster validation method using one-class support vector (SV) machine (OC-SVM)[5].

The proposed method consists of four major parts. They are:

- Preprocessing

- Principal component analysis

- Sub-pixel detection

- Fuzzy c - means

Section II of this paper describes the preprocessing technique which always must be done for any image processing. Preprocessing varies according to our requirement. This section gives various techniques and their advantages. Section III describes the principal component analysis technique. Principal components are the main components or features in an image. They are to be found out from the image in order to enable further processing efficiently. Here, preprocessing is done to reduce dimension of the high dimensional Hyperspectral data set. Section IV describes the subpixel target detection technique which is used to detect the target in the sub-pixel level. Various techniques used are also discussed. Section V discusses the Fuzzy c-mean algorithm which is used for segmentation of Hyperspectral image. Section VII consists of the results we obtained in each stage.

\section{PREPROCESSING:}

The aim of the spectral preprocessing is to avoid the effect of undesirable phenomena that affect the spectral measurement like light scattering, morphological differences or particle size effects such as surface roughness, detected artifacts etc. In the field of Hyperspectral the most general practice is to adapt well known preprocessing techniques of classical spectrography. 
Main spectral preprocessing techniques:

i) Denoising

ii) Scatter correction

iii) Derivatives

i) Denoising:

Denoising means the instrumental noise can be partially removed by using smoothing techniques. Many different algorithms are used in smoothing. But Savitzky-Golay is one of the well known techniques.

ii) Scatter correction:

Light scattering is one of the main pitfalls when for example, NIR spectra are measured. In the sense, the most widely used methods for scatter minimization are standard normal variate and multiplicative scattering correction.

iii) Derivatives:

The Savitzky-Golay filter is also used in finding spectral derivative in order to minimize additive effects as spectral base line slopes and offsets. First derivative eliminates an additive base line; second derivatives eliminate the linear base line. Generally, derivative transform emphasizes the spectral features of the data. But it can also promote on emphasis at the level of noise. So, special attention must be paid in the selection of the parameters for tuning the filter.

Standard A4 (210mm x 297mm) portrait page set-up should be used. The left, right, top and bottom margins should be $30 \mathrm{~mm}$. Do not use any headers, footers or footnotes. No page numbers. Single column. All main text paragraphs, including the abstract, must be fully (left and right) justified. All text, including title, authors, headings, captions and body, will be Times New Roman font.

\section{Principal COMPONENT ANAlysis:}

PCA is a widely used dimension reduction technique in image data analysis. It is the optimal linear scheme for minimizing a set of high dimensional vectors into a set of lower dimensional vectors[14]. There are two kinds of methods to perform Principal Component Analysis, the matrix method, and the data method. In this work, focus will be on matrix methods. To compute PCA, we follow the general 4 steps given below:

i) Find the mean vector in $x$-space.

ii) Assemble the covariance matrix in $x$-space.

iii) Compute Eigen values and corresponding eigenvectors.

iv) Form the components in y-space.

It is shown in the figure 1 that only the first few components are likely to contain the needed information. The number of components that hold the majority of or the very important information is called the intrinsic dimensionality and each data image may contain a different intrinsic dimensionality [7]. PCA condenses all the information of an "M" band original data set 
into a number of bands smaller than "M" (or principal components) in such a way that improves the covariance and reduces redundancy in order to achieve lower dimensionality but with rich information.

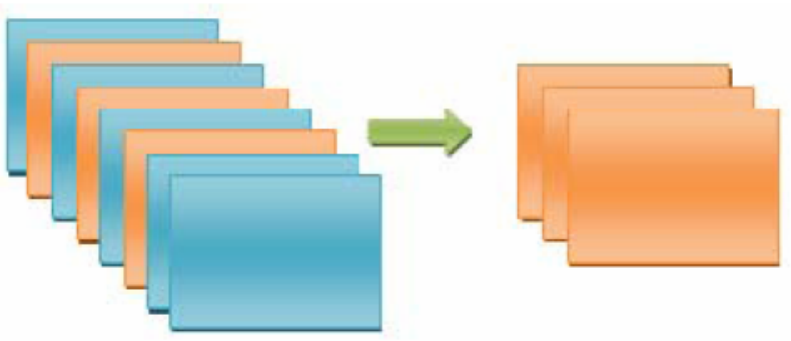

Figure 1. Dimensionality reduction using PCA

\section{SUB-PIXEL DETECTION TECHNIQUE:}

Sub-pixel analysis technique can be used for calculating the quantity of target materials in every pixel of an image. Sub-pixel analysis is able to detect quantities of the target that are much smaller than the pixel size itself. In any case of fine spectral contrast between a target and its background. Subpixel analysis has detected targets covering as small as 1- $3 \%$ of the pixel.

Sub-pixel analysis techniques include:

i) Complete linear spectral unmixing

ii) Matched filtering

iii) Mixture-tuned matched filtering

\section{i) Complete linear spectral unmixing:}

Any pixel spectral given is a linear combination of the spectra of all end members inside the pixels. Each end member weight is the proportion of area that the pixel contains the end member. Unmixing solves a set of a-linear equation for each pixel where ' $a$ ' is the number of bands in the image. The known variables in the given equations are the part of each end member in the pixel [15]. To be able to solve the linear equation for known pixel fractions, it is important to have known equations than unknowns. It means that more band end member materials are needed. With Hyperspectral images, this will almost always be true.

The outcomes of linear spectral unmixing include one abundance image for each end member [12]. The pixel value in such images indicates the percentage of the pixels which are made up of that end member. For instance, if any pixel in an abundance image for the end member quartz has a value of 0.80 then $80 \%$ of the area of the pixel does contain quartz. An error image is also calculated in order to evaluate the goodness of the unmixing analysis.

\section{ii) Matched filtering:}

It is a type of unmixing in which only 'user chosen targets' are mapped. Unlike in complete unmixing, it is not needed to know the spectra of all end members in the input to get an exact analysis. So, this kind of analysis is sometimes called a partial unmixing. The reason is that the unmixing equations can only be partially solved. 
Matched filtering filters the given input image for better matches to the chosen target spectrum by enhancing the response of the target spectrum within the data and neglecting the response of other spectrum expect the target one, which is treated as a composite unknown background to the target. Similar to complete unmixing, a pixel value in the response image is proportional to the fraction of the pixel which contains the target object. If a pixel contain a value of 0 or even lesser than with a value of 0 or less than it, then that will be interpreted as background.

One critical problem with this matched filtering technique is that, it may end up with false positive results. One possible solution to this problem that can be obtained in ENVI is to find out an additional measure called infeasibility that is the method called Mixture Tuned Matched Filtering.

\section{iii) Mixture tuned matched filtering:}

It is a hybrid method which is the combination of the matched filter method. It is not required to know all end members and linear mixture theory. The results of mixture tuned matched filtering will be two images. They are

a) MF score image (0-1)

b) Infeasibility image, the smaller its value better will be the matching.

Infeasibility is based on both noise and image statistics. This image indicates the degree to which the matched filtering outcome is a feasible mixture of the target and background. All pixels with high infeasibilities are likely to be false positives regardless of their matched filter value. 2D scatter plot is used to locate pixel in an image

\section{FUZZY CLUSTERING TECHNIQUE:}

Clustering of numerical data forms the basis of many classification and other system modeling algorithms. The main purpose of clustering is to identify natural groupings of data from a large data set to produce a concise representation of behavior of a system. FCM is a data clustering technique in which a dataset is grouped into $\mathrm{n}$ clusters with every data point in the dataset belonging to every cluster to a certain degree. For example, a certain data point that lies close to the center of a cluster will have a high degree of belonging or membership to that cluster and another data point that lies far away from the center of a cluster will have a low degree of belonging or membership to that cluster. It starts with an initial guess for the cluster centers, which are intended to mark the mean location of each and every cluster. The initial guess for such cluster centers is most likely to be incorrect. Next, FCM assigns every data point a membership grade for every cluster. By iteratively updating the cluster centers and the membership grades for each data point, FCM iteratively moves the cluster centers to the right location within a particular data set. This iteration is based on reducing an objective function that represents the distance from any given data point to a cluster center weighted by that data point's membership grade.

The aim of image segmentation is to partition an image into a set of disjoint regions with uniform and homogeneous attributes such as intensity, color, tone or texture, etc. Many segmentation techniques have been developed and detailed surveys can be found in references. The image segmentation approaches can be divided into four categories. They are Thresholding, clustering, edge detection and region extraction. Clustering is a process for classifying objects or patterns in such a way that samples of the same group are more similar to one another than samples belonging to various groups. Many clustering strategies have been used, such as the hard 
clustering scheme and the fuzzy clustering technique, each of which has its own special characteristics. . Among the fuzzy clustering methods, FCM algorithm is the most popular method used in image segmentation because it has robust characteristics for ambiguity and can retain more information than hard segmentation methods.

The FCM clustering algorithm was first introduced by Dunn and later was extended by Bezdek. The algorithm is an iterative clustering method that produces an optimal c partition by minimizing the weighted within group sum of squared error objective function $\mathrm{J}_{\mathrm{FCM}}$ :

$$
J_{F C M}=\sum_{k=1}^{n} \sum_{i=1}^{c}\left(u_{i k}\right)^{q} d^{2}\left(x_{k}, v_{i}\right)
$$

where $X=\{x 1, x 2, \cdots, x n\} \subseteq R p$ is the data set in the $p$-dimensional vector space, $n$ is the number of data items, $c$ is the number of clusters with $2 \leq \mathrm{c}<\mathrm{n}, \mathrm{u}_{\mathrm{ik}}$ is the degree of membership of $x_{k}$ in the $i^{\text {th }}$ cluster, $q$ is a weighting exponent on each fuzzy membership, vi is the prototype of the centre of cluster $i, d^{2}\left(x_{k}, v i\right)$ is a distance measure between object $x_{k}$ and cluster centre vi. A solution of the object function $\mathrm{J}_{\mathrm{FCM}}$ can be obtained via an iterative process, which is carried out as follows:

1. Set values for $\mathrm{c}, \mathrm{q}$ and $\mathrm{Q}$.

2. Initialize the fuzzy partition matrix $U=\left[\mathrm{u}_{\mathrm{ik}}\right]$.

3. Set the loop counter $b=0$.

4. Calculate the c cluster centers

$$
v_{i}{ }^{(c)}=\frac{\sum_{k=1}^{n}\left(u_{i k}{ }^{(c)}\right)^{q} x_{k}}{\sum_{k=1}^{n}\left(u_{i k}{ }^{(c)}\right)^{q}}
$$

5. Calculate the membership $\mathrm{U}(\mathrm{b}+1)$. For $\mathrm{k}=1$ to $\mathrm{n}$, calculate the following:

$I_{k}=\left\{i \mid 1 \leq i \leq c, d_{i k}=\left\|x_{k}-v_{i}\right\|=0\right\}, / \mathrm{I}$ for the $\mathrm{k}^{\text {th }}$ column of the matrix, compute new membership values:

(a) if $\mathrm{Ik}=\varphi$, then

$$
U_{i k}^{c+1}=\frac{1}{\sum_{j=1}^{c}\left(\left(\frac{d i_{k}}{d j_{k}}\right)^{\frac{2}{q-1}}\right)}
$$

(b)Else $U_{i k}^{(c+1)}=0$ for all $i \nexists I$ and $\sum i \varepsilon I_{k} U_{i k}^{(c+1)}=1$; next $k$

6. If $\left\|U^{(c)}-U^{(c+1)}\right\|<\mathrm{e}$, stop; otherwise, set $\mathrm{c}=\mathrm{c}+1$ and go to step 4 .

\section{PROPOSED WORK:}

The main objective is to select a particular band of the entire hyperspectral dataset. As a first step preprocessing is done in order to remove the noise present in the input band. Salt and pepper noise present in the image is removed by using median filter. Then principal component analysis has been performed in order to reduce the dimension of the Hyperspectral image. As a result of PCA only few principal components of the image is obtained. The result of principal component 
analysis is then further used in sub-pixel target detection. Then fuzzy c-means algorithm is used to obtain the segmented output.

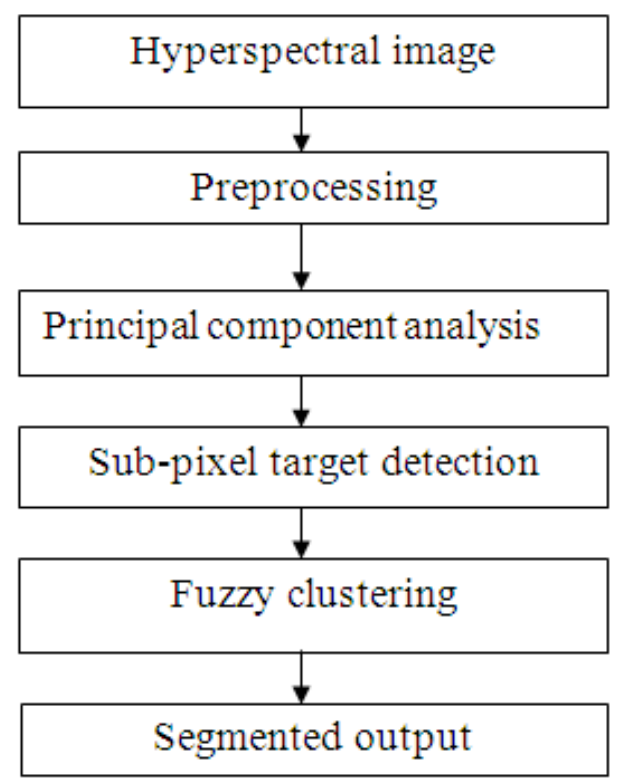

Fig. 7.1 Flow diagram of the proposed work

\section{EXPERIMENTAL OUTPUT:}

The image we have taken is from the datasets of Indian pines captured by AVIRIS sensor. This Indian pines dataset contain 220 contiguous bands. At a time, all the bands cannot be loaded. To further process the Hyperspectral image a particular band should be selected and the high dimensional data should be reduced to smaller one. Figure 3 represents the $100^{\text {th }}$ band of the Hyperspectral image "Indian_pines".

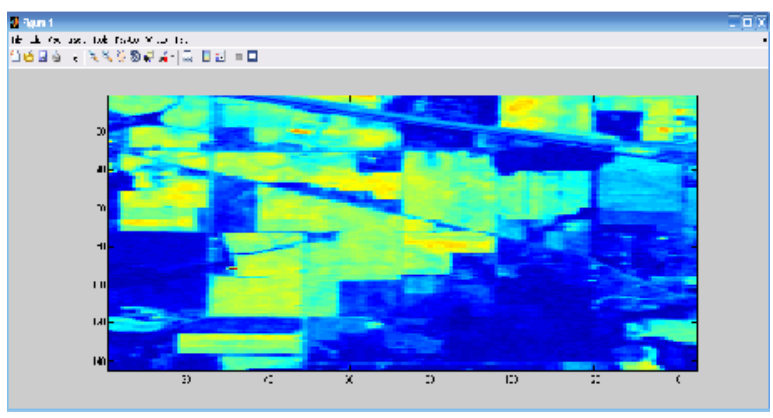

Figure $3.100^{\text {th }}$ band of Indian_pines, AVIRIS.

Figure 4 represents preprocessed output of $100^{\text {th }}$ band of the input Hyperspectral image. For preprocessing median filter is used. This output will be out of noises in the image mainly salt and pepper noise. 


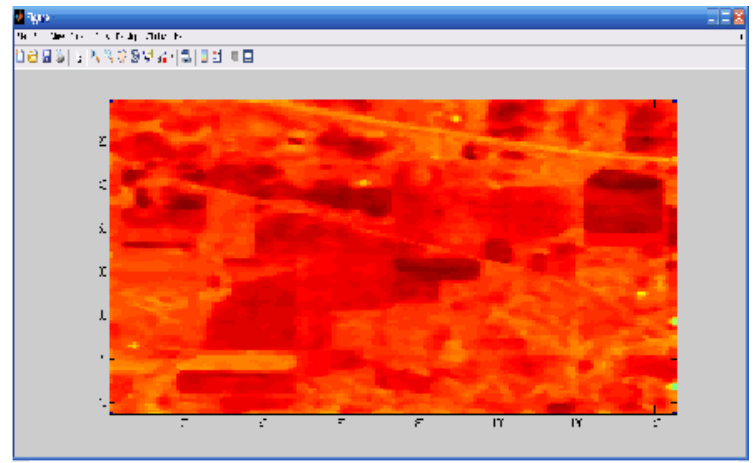

Figure 4. Preprocessed image

Figure 5 represents the ground truth image of Indian_pines. This ground truth image consists of information collected on particular location by the sensor.

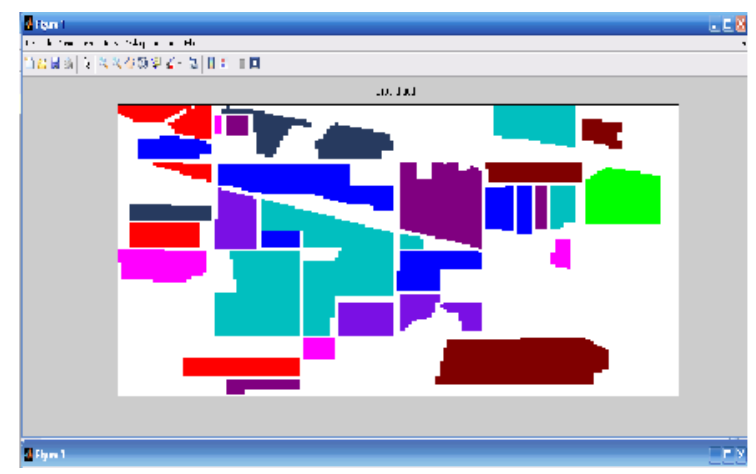

Figure 5. Ground truth image

Figure 6 represents the output obtained on applying the fuzzy clustering algorithm on the output of the matched filtering technique. Overall accuracy is defined as the ratio of sum of all pixel which are in agreement between ground truth image and segmented output to the total no. of pixels in the ground truth image.

$$
\text { overall accuracy }=\frac{\text { No. of pixels in agreement }}{\text { Total no. of pixels }}
$$

Also the overall accuracy of the segmented output is found as 0.94682. This shows better accuracy compared to the outputs obtained from the previous segmentation techniques. 


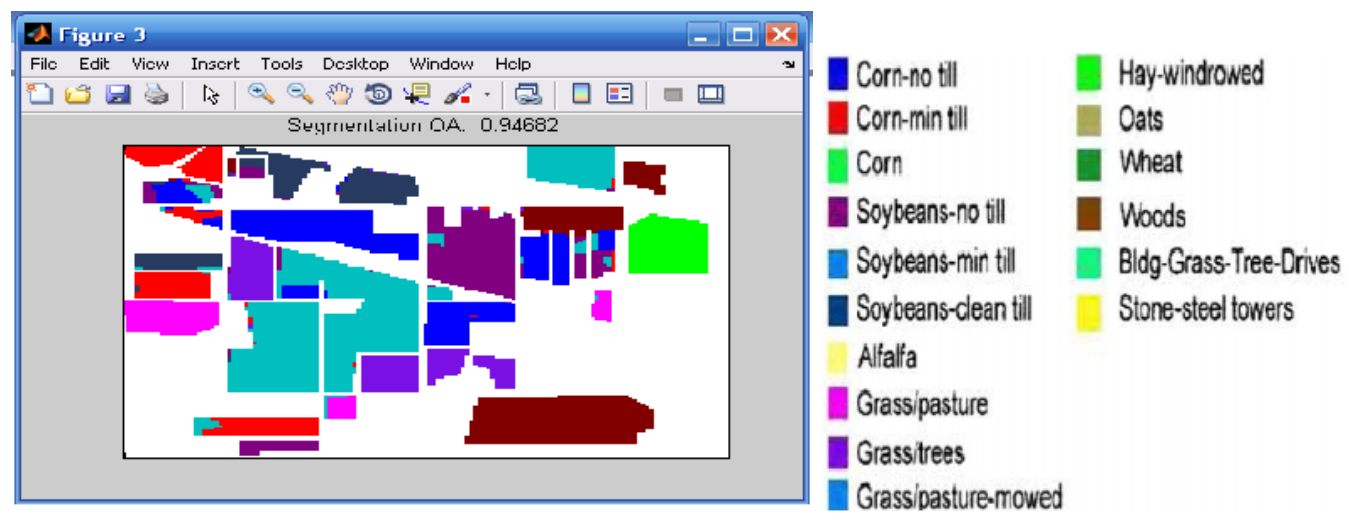

Figure 6 Segmented output

\section{CONCLUSION:}

Thus a new Hyperspectral image segmentation technique is proposed which combines sub-pixel detection and fuzzy clustering technique. The number of bands is reduced by using principal component analysis technique. Only useful bands were selected and segmentation was done based on the values obtained using principal component analysis. Since fuzzy c-means is used, the clustering becomes simple. The overall accuracy is found to be 0.94682 .

\section{REFERENCES}

[1] Jun Li,José M.Bioucas-Dias and Antonio Plaza, "Spectral-spatial Hyperspectral Image Segmentation Using Subspace Multinomial Logistic Regression and Markov Random Fields", 2012, ieee transactions on geoscience and remote sensing, vol. 50, no. 3, march 2012.

[2] Jun Li, José M.Bioucas-Dias and Antonio Plaza "Hyperspectral Image Segmentation Using a New Bayesian Approach With Active Learning",2011, ieee transactions on geoscience and remote sensing, vol. 49, no. 10, october 2011.

[3] Janete S. Borges, José M. Bioucas-Dias and Andre R. S. Marcal. “ Bayesian Hyperspectral Image Segmentation With Discriminative ClassLearning",2011, ieee transactions on geoscience and remote sensing, vol. 49, no. 6, june 2011.

[4] Hernâni Gonçalves, Luís Corte-Real, and José A. Gonçalves ‘Automatic Image Registration Through Image Segmentation and SIFT', ,2011, ieee transactions on geoscience and remote sensing, vol. 49, no. 7, july 2011.

[5] Gökhan Bilgin, Sarp Ertürk, Member, IEEE, and Tülay Yıldırım, "Segmentation of Hyperspectral Images via Subtractive Clustering and Cluster Validation Using One-Class Support Vector Machines",2011. ieee transactions on geoscience and remote sensing, vol. 49, no. 8, august 2011.

[6] J. Silverman, S. R. Rotman, and C. E. Caefer, "Segmentation of hyperspectral images based on histograms of principal components," in Proc. SPIE, 2002, vol. 4816, pp. 270-277.

[7] N. Acito, G. Corsini, and M. Diani, "An unsupervised algorithm for hyperspectral image segmentation based on the Gaussian mixture model," in Proc. IEEE IGARSS, Toulouse, France, Jul. 2003, vol. 6, pp. 3745-3747.

[8] M. D. Farrell, Jr. and R. Mersereau, "Robust automatic clustering of hyperspectral imagery using nonGaussian mixtures,” in Proc. SPIE, 2004, vol. 5573, pp. 161-172.

[9] G. Mercier, S. Derrode, and M. Lennon, "Hyperspectral image segmentation with Markov chain model," in Proc. IEEE Geosci. Remote Sens. Symp., Toulouse, France, Jul. 2003, vol. 6, pp. 37663768.

[10] H. H. Muhammed, "Unsupervised hyperspectral image segmentation using a new class of neurofuzzy systems based on weighted incremental neural networks," in Proc. IEEE 31st Appl. Image Pattern Recognit. Workshop, Washington, DC, Oct. 2002, pp. 171-177. 
International Journal of Information Sciences and Techniques (IJIST) Vol.4, No.3, May 2014

[11] J. S. Beis and D. G. Lowe, "Shape indexing using approximate nearestneighbor search in highdimensional spaces,” in Proc. Conf. Comput. Vis. Pattern Recog., Washington, DC, 1997, pp. 10001006.

[12] L. G. Brown, "A survey of image registration techniques," Comput. Surv., vol. 24, no. 4, pp. 325376, Dec. 1992.

[13] H. D. Cheng, X. H. Jiang, Y. Sun, and J. Wang, "Color image segmentation: Advances and prospects," Pattern Recog., vol. 34, no. 12, pp. 2259-2281, Dec. 2001.

[14] L. Cheng, J. Gong, X. Yang, C. Fan, and P. Han, "Robust affine invariant feature extraction for image matching," IEEE Geosci. Remote Sens. Lett., vol. 5, no. 2, pp. 246-250, Apr. 2008.

[15] P. Dare and I. Dowman, "An improved model for automatic feature-based registration of SAR and SPOT images,” Proc. ISPRS J. Photogramm. Remote Sens., vol. 56, no. 1, pp. 13-28, Jun. 2001. 